\title{
An Efficient Frequency-Domain Analysis Technique of MOSFET Operation
}

\author{
Kyu-Il Lee, Jinsoo Kim, *Hyungsoon Shin, **Chanho Lee, Young June Park,
} and Hong Shick Min

School of EE and CS, Seoul National Univ., Seoul, Korea, E-mail : macjr@bawi.org

*Dept. of IEE, Ewha Womans Univ., Seoul, Korea

**School Of EE, Soongsil Univ., Seoul, Korea

\begin{abstract}
We propose a harmonic balance technique for the frequency-domain analysis of MOSFET operation. Our approach is based on the charge-sheet and the non-quasistatic(NQS) MOSFET models in the channel region with the harmonic balance $(\mathrm{HB})$ technique applied to the channel charges. Lateral field effect is considered in the formulation to analyze the short channel MOSFET devices. It is shown that the proposed method renders a computationally efficient tool to analyze the harmonic distortion occurrence in the MOSFET devices due to the nonlinear response of the channel charges.
\end{abstract}

\section{Introduction}

The modeling of the distortion in the MOSFET under the multi-tone input signal becomes an important issue as the CMOS RF circuit technology is merged as a main stream in recent RF technology. The conventional time-domain device simulation is not adequate for this purpose since too many integration steps are required to trace the multi-tone signal response[1]. Hence, the frequency-domain approaches including harmonic balance technique have been used in the two-dimensional numerical simulators to cope with the difficulties. But, as can be known from PISCES-HB[2], the numerical burden in HB analysis becomes extremely heavy, which may cause efficiency problem in the practical applications.

Aiming at an efficient RF device simulation, we have proposed a new HB method[3] based on the charge-sheet[4] and the non-quasistatic[5] MOSFET models with longchannel approximation, which greatly reduces numerical burden but gives most of the essential features of the harmonic behavior of MOSFET. In this paper, the method to extend the scheme to the short channel device will be proposed.

\section{Frequency-Domain Formulation of MOSFET Model}

We employ a one-dimensional nMOSFET model as the basic framework of our method, which describes the normalized charge density at a location $x$ in the channel and a time $t$ as[5]

$$
\begin{aligned}
q_{e}(y, t) & =V_{G B}(t)-V_{F B}-\phi_{S}(y, t)-\gamma \sqrt{\phi_{S}(y, t)-V_{t}} \\
& \cong V_{G B}(t)-V_{F B}-\phi_{S 0}(t)-\gamma \sqrt{\phi_{S 0}(t)-V_{t}}-F_{B}(t) \cdot\left\{\phi_{S}(y, t)-\phi_{S 0}(t)\right\} .
\end{aligned}
$$

For numerical approach, boundary conditions $\phi_{s 0}(t)$ and $\phi_{s L}(t)$ (surface potentials at both ends of the channel) are calculated from the MOSFET charge equation. Consequently, the current continuity equation can be written as

$$
\frac{\partial q_{e}(y, t)}{\partial t}=\frac{\mu_{e}}{2 F_{B}(t)} \cdot \frac{\partial^{2}}{\partial y^{2}}\left[\left\{q_{e}(y, t)+F_{B}(t) \cdot V_{t}\right\}^{2}\right]
$$

where the electron mobility $\mu_{e}$ is assumed to be a constant value of $500\left[\mathrm{~cm}^{2} / \mathrm{V} \cdot \mathrm{sec}\right]$. Our goal is to find a numerical approximant $q_{e}$ satisfying Eq. (2) for given boundary conditions. Instead of time-domain treatment, we pose the problem in frequency 
domain dual to time or space variable and consider the corresponding numerical scheme together with the effect of the NQS component.

Pursuing Park's work[6], we can decompose the normalized charge density into two parts, the quasistatic and the non-quasistatic components:

$q_{e}(y, t)=q_{e}^{Q S}(y, t)+q_{e}^{N Q S}(y, t)$.

The quasistatic component $q_{e}^{Q s}$ is obtained analytically by setting the left-hand-sided time-derivative term in Eq. (2) to be zero. Let us assume that the inversion charges at both source and drain ends of the channel respond instantaneously according to the changes of biases, the non-quasistatic component $q_{e}^{N Q S}$ can then be approximated by finite Fourier sine series as

$$
q_{e}^{N Q S}(y, t)=\sum_{m=1}^{M} Q_{m}(t) \cdot \sin \left(\frac{m \pi y}{L}\right) \text { and } Q_{m}(t)=Q_{m, 0}+\sum_{h=1}^{H}\left\{Q_{m, h}^{R} \cos \left(\omega_{h} t\right)-Q_{m, h}^{I} \sin \left(\omega_{h} t\right)\right\} \text {. }
$$

The Fourier coefficients of $Q_{m}(t)$ compose our solution vector. With the above equations, the least-squared solution of the nonlinear system from Eq. (2) is obtained by Newton-Raphson method via $(2 H+l)$ time and $M$ space samples. For the comparison purpose in terms of the accuracy and computational efficiency, we also follow the finite-difference method (FDM) adopted in the usual harmonic balance simulation tools and Park's mathematical approximation for SPICE.

For the modeling of the short channel effect, the lateral field effect has to be considered in the formulation[7]. Thus the model equations are changed to include lateral field term which contains the first and second derivatives of the surface potential. For the numerical approach, we assume the linear relationship between the potential and the charge density and the averaged value of the first and second derivatives of the surface potential are used in the numerical scheme. The resulting form of the master equation for iteration is the same as Eq. (2) except for the modification in the correct factor $F_{B}$. Thus the numerical complexity and burden are unchanged compared to the long channel formulation. Further consideration of the short channel effect is under development.

\section{Results and Discussion}

Device and bias conditions for simulation are shown in Table 1.

Figures 1 and 2 compare the source and drain currents obtained by our method, FDM, Park's approach, and MEDICI for rising and falling transitions of ramp input signal on $10[\mu \mathrm{m}]$ MOSFET, where a general agreement is observed except small deviation caused by the slight differences in physical parameters. The peak values of the NQS charge density at each node are depicted in Fig. 3 to show the chargetransit time effect. In the turn-on stage, the absolute value of the NQS component becomes larger toward the drain end due to the charge-transit time. In the turn-on stage, as the inversion charges are supplied from the source, the charges near drain end cannot be induced quickly according to the bias change. In the turn-off stage, however, the NQS component is not maximal in the vicinity of the channel ends because the inversion charges remote from both ends of the channel suffer the propagation delay. Since boundary potentials at both ends are not identical, this phenomenon is not symmetric around the center of the channel. However, FDM using 10 space nodes in the present example requires longer simulation time than the NQS methods. The simulation of the short channel devices using FDM requires fine discretization and more nodes in the channel(especially near junction) at the expense of simulation efficiency as the charge density changes drastically in the channel. 
Table 1. Device parameters and bias conditions for simulation

\begin{tabular}{|c|c|c|c|c|}
\hline & $\mathbf{N}_{\mathbf{A}}$ & $\mathbf{N}_{\mathbf{D}}$ & $\mathbf{T}_{\mathbf{O X}}$ & $\mathbf{V}_{\mathbf{D S}}$ \\
\hline $\mathbf{L}=\mathbf{1 0}[\boldsymbol{\mu m}]$ & $2.1 \times 10^{16}\left[\mathrm{~cm}^{-3}\right]$ & & $180[\AA]$ & \multirow{2}{*}{$1[\mathrm{~V}]$} \\
\cline { 1 - 2 } $\mathbf{L}=\mathbf{5}[\mu \mathrm{m}]$ & $5.0 \times 10^{16}\left[\mathrm{~cm}^{-3}\right]$ & $5.0 \times 10^{20}\left[\mathrm{~cm}^{-3}\right]$ & $150[\AA]$ & \\
\cline { 1 - 3 } $\mathbf{L}=\mathbf{0 . 5}[\mu \mathrm{m}]$ & $2.0 \times 10^{17}\left[\mathrm{~cm}^{-3}\right]$ & & $80[\AA$ & $5[\mathrm{~V}]$ \\
\hline Ramp & From $0[\mathrm{~V}]$ to $5[\mathrm{~V}]$, rising \& falling time $=2[\mathrm{nsec}], 20[\mathrm{psec}]$ \\
\hline Single-tone & \multicolumn{3}{|c|}{$V_{g b}=3+1 \cdot \sin \left(2 \pi f_{1} t\right)[\mathrm{V}]$} \\
\hline Two-tone & \multicolumn{2}{|c|}{$V_{g b}=3+1 \cdot \sin \left(2 \pi f_{1} t\right)+1 \cdot \sin \left(2 \pi f_{2} t\right)[\mathrm{V}]$} \\
\hline
\end{tabular}

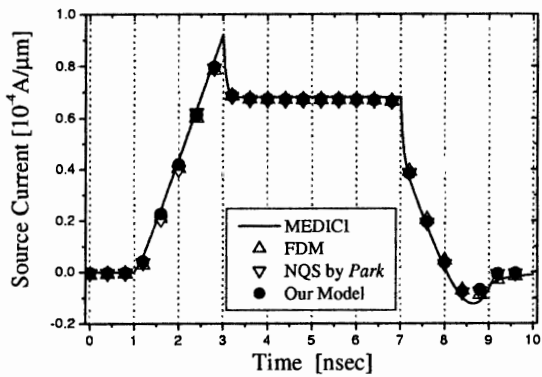

Fig. 1. Source currents of the $10[\mu \mathrm{m}]$ MOSFET for ramp input

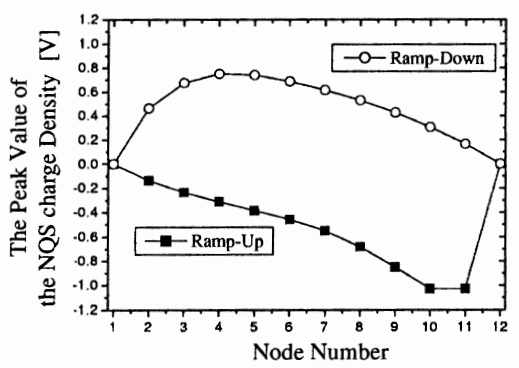

Fig. 3 Peak value of the NQS charges in the channel (1: Source, 12 : Drain)

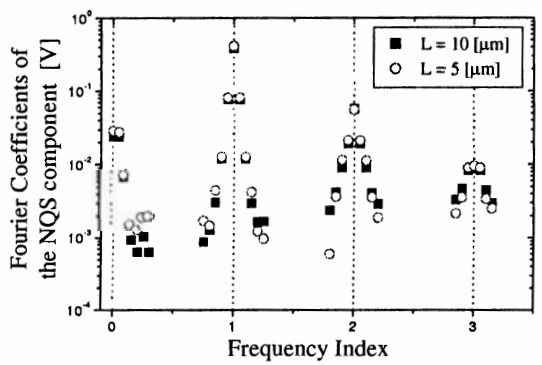

Fig. 5 Fourier Coefficients of the NQS component for two-tone input

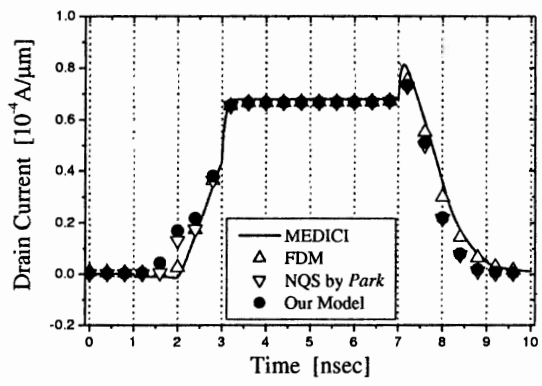

Fig. 2 Drain currents of the $10[\mu \mathrm{m}]$ MOSFET for ramp input

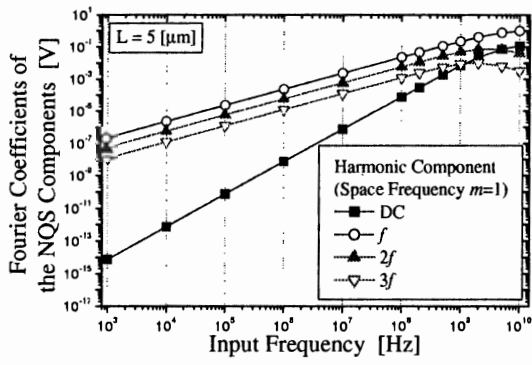

Fig. 4 Fourier Coefficients of the NQS component for single-tone input

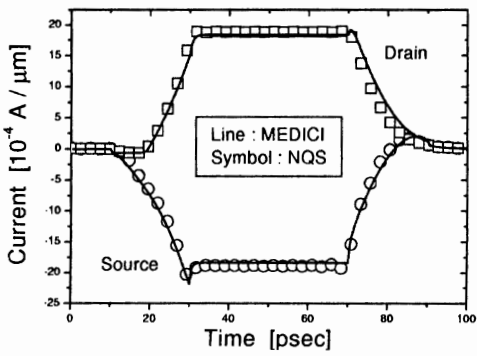

Fig. 6 Currents of the $0.5[\mu \mathrm{m}]$ MOSFET for ramp input 
In Fig. $4(\mathrm{~L}=5[\mu \mathrm{m}])$, the DC and the first three terms of Eq. (5) are shown for the case of space frequency $m=1$, which are the dominant terms of $q_{e}$. When input frequencies are higher than the intrinsic cut-off frequency, the NQS components are responsible for harmonic distortion. It has been reported that the third-order harmonic distortion component shows the notch behavior[8]. The value of the third harmonic Fourier cosine coefficient changes from positive to negative and again to positive as the input frequency increases. The change in sign causes the notch behavior in the harmonic distortion.

In Fig. 5, the Fourier coefficients of the NQS charges in case of two-tone input on gate are shown for four dominant harmonic components of $f_{2}$ (the higher frequency, $100[\mathrm{MHz}]$ ), around each of which has several harmonic components of $f_{1}$ (the lower frequency, $10[\mathrm{kHz}])$. These harmonic and intermodulated components will give rise to the nonlinear distortion to the output currents. However, two-tone large-signal input signal in this example is not tractable in most device simulation tools.

Figure 6 compares the current of $0.5[\mu \mathrm{m}]$ MOSFET in the saturation region $\left(V_{D}=5[\mathrm{~V}]\right)$ with the result of MEDICI. The current values from our method are consistent with those from MEDICI when the MOSFET is operated in the saturation condition(high lateral field). This example confirms the applicability of the modification in the correction factor $F_{B}$ for the short channel MOSFET.

\section{Conclusion}

It is shown that our simple but novel and physically reasonable method has many merits over the conventional approaches in the analysis of AC signals in view of the numerical burden; computation time and memory usage. It is also shown that the simple modification on the model is acceptable in analyzing the short channel effect and further consideration of the short channel effect is under development. The error that may be caused by the simplification of the model formulation is compensated by the non-quasistatic treatment. Also the proposed model can be extended to the direct combination of the device simulation with the circuit simulation.

\section{Acknowledgment}

This work has been supported by the National Research Laboratory Project of the Ministry of Science and Technology and by the Brain Korea 21 Project.

\section{References}

[1] Kevenaar, T. A., and Maten, E. J. W. ter (1999), RF IC Simulation : state-of-the-art and future tends, SISPAD, pp.7-10

[2] Troyanovsky, B., Yu, Z., Yu, and Dutton, R. W. (1995), Large Signal Frequency Domain Device Analysis via the Harmonic Balance Technique, SISPAD, pp.114-117

[3] Lee, K. -I., Kim, J., Park, Y. J., and Min, H. S., A Simple Frequency-Domain Analysis of MOSFET - Including Non-Quasistatic Effect, IEEE TCAD, To-be-published in July 2001

[4] Brews, J. R. (1978) : A Charge-sheet Model of the MOSFET, SSE vol.21, pp.345-355

[5] Park, H. J. (1989) : Charge-sheet and Non-quasistatic MOSFET Models for SPICE, Ph. D. Dissertation, U. C. Berkeley

[6] Park, H. J., Ko, P. K., and Hu, C. (1986) : A Measurement-based Charge-sheet capacitance Model of Short-channel MOSFET's for SPICE, IEDM, pp.40- 43

[7] Miura-Mattausch, M., Feldmann, U., Rahm, A., Bollu, M., and Savignac, D. (1996) : Unified Complete MOSFET Model for Analysis of Digital and Analog Circuits, IEEE TCAD vol. 15, pp. 1-7

[8] Pu, L. -J., and Tsividis, Y. P. (1990) : Harmonic Distortion of the Four-terminal MOSFET in Non-quasistatic Operation, IEE Proceedings vol. 137, pp.325-332 